\title{
Simulieren was selten geschieht. Nutzerzentrierte Entwicklung mobiler Simulatoren für komplexe maritime Großschadenslagen
}

\author{
Patrick Roßner ${ }^{1}$ Max Bernhagen ${ }^{1} \cdot$ Angelika C. Bullinger ${ }^{1}$ \\ Online publiziert: 15 . Juli 2020 \\ (c) Der/die Autor(en) 2020
}

Schlüsselwörter Nutzerzentrierter Entwicklungsprozess · Simulation · Virtuelle Prototypen · Maritime Großschadenslagen

\section{Einleitung}

Großschadensereignisse geschehen selten, gehen aber mit einer erheblichen Gefahr für Personen und Sachgüter sowie für die Sicherheit von Schifffahrtswegen einher und erfordern daher die besondere Kompetenz aller am Rettungswesen beteiligten Organisationen. Gleichzeitig stellen derartige Ereignisse aufgrund der Komplexität der Situation (u.a. Art des Schiffstyps, Art der Ladung, Anzahl $\mathrm{zu}$ bergender Menschen, Auswirkungen auf die Umwelt) und der großen Zahl beteiligter Organisationen (z. B. Deutsche Gesellschaft zur Rettung Schiffbrüchiger, Feuerwehr, Bundespolizei, Technisches Hilfswerk, Luftrettung) einzigartige Ansprüche an alle Beteiligten. Ereignisse wie der Brand auf der Fähre Lisco Gloria im Oktober 2010 in deutsch-dänischen Gewässern zeigen zudem die Bedeutung der reibungslosen Zusammenarbeit internationaler Hilfsorganisationen (Rosenkötter 2015). Die Havarie der „Costa Concordia“ im Januar 2012 vor Giglio ist zudem ein Beispiel für die Dimension möglicher Rettungseinsätze auf See (Schlamp 2018), die sich aber auch auf Binnengewässern Kollision der Viking Freya mit einer Brücke - und in Häfen - Brand des Containerschiffes CCNI Arauco - zeigen (Buchenau 2020).

Zur Rettung entwickeln sich in den jeweils beteiligten Organisationen spontane Ad-hoc-Organisationsstrukturen, um anfallende Aufgaben zu bewältigen. Dabei können vorhandene Organisationskulturen, verschiedene Einsatzrollen und eine Vielzahl weiterer Faktoren zu einer unterschied-

Patrick Roßner

patrick.rossner@mb.tu-chemnitz.de

1 Technische Universität Chemnitz, Professur Arbeitswissenschaft und Innovationsmanagement, Erfenschlager Straße 73, Gebäude C, 09125 Chemnitz, Sachsen, Deutschland lichen Interpretation der Lage führen. Unterscheiden sich die Interpretationen zwischen den beteiligten Organisationen, verringert sich die Effizienz und Effektivität der Kommunikation und Missverständnisse werden wahrscheinlich (Salas et al. 2006; Flin et al. 2008; Zoller 2014). Aus diesem Grund ist regelmäßiges gemeinsames Training der (potentiell) beteiligten Organisationen nicht nur zur Verbesserung der zur Krisenbewältigung benötigten Grundfertigkeiten elementar (Strohschneider 2012; Tena-Chollet et al. 2016). Das Training verbessert auch eine der Situation angemessene Handlungsreaktion und ein gruppenspezifisches Problemlösen (Weick 2010, Hofinger 2008).

Diesem Bedarf an Training mit möglichst vielen beteiligten Organisationen, häufig auch über Landesgrenzen hinweg, steht der hohe finanzielle und organisatorische Aufwand von realitätsnahen Übungen solcher Situationen gegenüber. Eine inhaltlich und ökonomisch sinnvolle Alternative wäre das Training im Simulator. Simulatorübungen sind weit kostengünstiger als Realweltübungen und können mit geringem zeitlichem Aufwand flexibel eingesetzt werden (Wright und Madey 2008; Molka-Danielsen et al. 2018). Jedoch sind derzeit für maritime Großschadenslagen vorrangig Insellösungen verfügbar, die keine organisationsübergreifenden Trainingsszenarien zulassen und damit nicht zur Simulation geeignet sind. Es besteht Bedarf an modularen, vernetzten Simulatoren, die mit einem zentralen Instruktionszentrum verbunden sind (Riener 2010). Darüber hinaus fehlen Simulatoren für mobile, modulare und multifunktionale Operationsstationen (MOS - Mobile Operation Station), die beispielsweise einen Schiffsführer-, Helikopter- und Leitstellenarbeitsplatz simulieren und somit die Breite der Tätigkeiten der Rettungskräfte bei einer maritimen Großschadenslage abbilden können (Lübcke et al. 2015).

Der Beitrag stellt die nutzerzentrierte Entwicklung und Evaluation der MOS mithilfe des nutzerzentrierten Ent- 
wicklungsprozesses dar (International Organization for Standardization (ISO) 2010). Auf Basis von Fokusgruppen mit späteren Nutzergruppen wurden anwendungsbezogene Anforderungen erhoben, ein Gestaltungskonzept entworfen und dieses in drei Iterationen kontinuierlich weiterentwickelt. Anschließend erfolgte eine Evaluation durch Nutzertests mit quantitativen und qualitativen Daten, deren Ergebnisse die Basis für die finale Anpassung darstellten. Am Ende des Entwicklungsprozesses steht ein Konzept der MOS, dass die Anforderungen aller Nutzergruppen erfüllt und eine hohe Gebrauchstauglichkeit für alle drei Arbeitsplätze aufweist. Im Ausblick werden die gewonnenen Erkenntnisse für die Entwicklung derart komplexer Simulatoren zusammengefasst und aufgezeigt, welche Komponenten von besonderer Bedeutung für das Simulieren und Trainieren von Großschadensereignissen sind. Zudem werden Vor- und Nachteile der angewandten Methoden diskutiert.

\section{Nutzerzentrierte Entwicklung der MOS}

Die MOS muss drei unterschiedliche Anwendungsfälle bedienen und somit in der Lage sein, die Arbeitsumgebungen eines Schiffsführers, Helikopterpiloten und Leitstellenmitarbeiters abzubilden. Dafür muss ein hinreichend generisches Design basierend auf den Nutzeranforderungen entwickelt werden, das realistische Workloadlevels im Simulatortraining reproduziert, ohne eine hochgradig detailgetreue Umgebung (siehe 2.3.1) zur Verfügung zu stellen. Gleichzeitig stellt die erforderliche Mobilität und Modularität groBe Herausforderungen an die zu verwendeten Materialien und Montagemethoden. Ohne großen Aufwand und ohne fachspezifische Kenntnisse im Bereich Soft- und Hardwareinstallation sollte es jedem potentiellen Nutzer möglich sein, die MOS auf- und umzubauen sowie in Betrieb zu nehmen.

Entwicklungsaktivitäten der Vergangenheit konzentrierten sich zumeist auf die funktionale Produktentwicklung (Schlick et al. 2010), wobei vor allem finanzielle und technologische Anforderungen bei der Entwicklung eines neuen Produkts im Fokus standen. Der nutzerzentrierte Entwicklungsprozess (UCD, siehe Abb. 1) bezieht Personen bzw. Nutzergruppen, die das System später verwenden, schon in frühen Projektphasen in die Entwicklung ein und verbessert die Gebrauchstauglichkeit der entwickelten Produkte (ISO 2010). Um hohen Usability- und Ergonomieanforderungen von heterogenen Nutzergruppen gerecht zu werden, stellt der UCD eine sehr gut geeignete und präzise Methode dar (Bevan 2009) und wird in vielen verschiedenen Domänen eingesetzt, bspw. auch zur Ausgestaltung von Lehrinhalten (Kahraman 2010; Ecker 2016). Zu den Vorteilen zählen darüber hinaus sicher bedienbare Produkte, auf lange Sicht

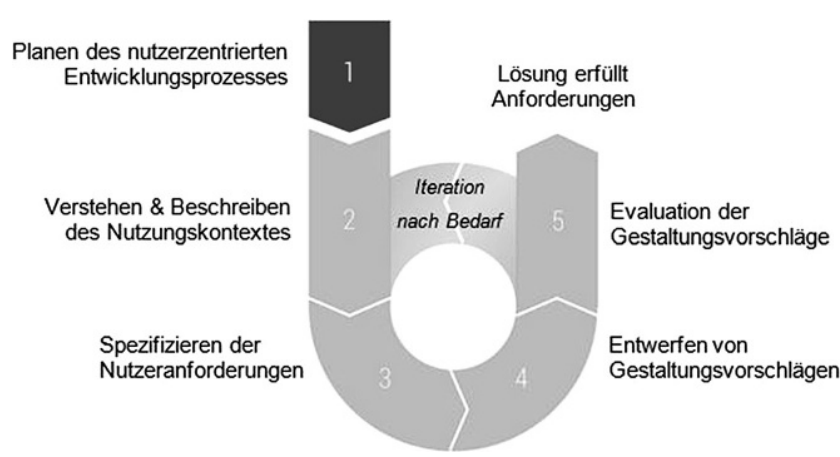

Abb. 1 Vorgehen des nutzerzentrierten Entwicklungsprozesses (ISO $9241-210)$

geringere Wartungs- und Betriebskosten (Ritter et al. 2014) und reduzierte Trainingsaufwände für die MOS (ISO 2010; Chammas et al. 2015).

Der nutzerzentrierte Entwicklungsprozess ist unterteilt in fünf Phasen. Nach einer allgemeinen Planungsphase müssen Entwickler weitere vier Phasen durchlaufen, beginnend mit der Analyse des Nutzungskontexts. In dieser Phase wird die Charakterisierung der Nutzer anhand ihrer Aufgaben, ihres physischen und sozialen Umfelds sowie ihrer Arbeits- und Hilfsmittel vorgenommen. In der folgenden Phase müssen die Informationen des Nutzungskontextes verfeinert werden, um Anforderungen für das zu entwickelnde Produkt abzuleiten. Methoden, die die Spezifikation von Anforderungen unterstützen, sind Personas oder Anwendungsszenarien (Maguire 2001). Die Erstellung von Designlösungen ist die Hauptaufgabe während der dritten Phase. Entwickler erstellen unter Verwendung von Methoden wie Paper Prototyping (Bailey et al. 2008, Still und Morris 2010) oder nach genormten Richtlinien und Standards (bspw. ISO 9241 - 110) mehrere Design-alternativen. In dieser Phase müssen Entwickler sowohl die technischen als auch die Anforderungen, die sich aus dem Nutzungskontext ergeben, berücksichtigen. In der vierten Phase bewerten Anwender oder Experten die erstellten Designlösungen entsprechend den vorher definierten Anforderungen, z.B. mittels Standard Review (Nielsen 1994, Shneiderman et al. 2016) oder Cognitive Walkthrough (Blackmon 2004). Entwicklern wird somit die Möglichkeit geboten, bereits in frühen Entwicklungsstadien von einer Bewertung der Designlösungen zu profitieren, mögliche Schwachstellen zu identifizieren und in weiteren Iterationsschleifen Verbesserungen zu implementieren. Im Folgenden wird der nutzerzentrierte Entwicklungsprozess für die mobile, modulare und multifunktionale Operationsstation beschrieben.

\subsection{Nutzungskontextanalyse}

Für die Umsetzung der ersten Phase des nutzerzentrierten Entwicklungsprozesses, der Analyse des Nutzungskontex- 
tes der MOS, wurde ein Methoden-Mix gewählt (Creswell 2009), der im Ergebnis qualitative und quantitative Daten kombiniert und sinnvollerweise in, von den beteiligten Forschern, unbekannten Anwendungsbereichen eingesetzt wird. Um die Bedarfe an Anzeige- und Bedienelementen sowie Simulationsparametern aller Nutzergruppen (Schiffsführer, Helikopterpiloten und Leitstellenmitarbeiter) und der daraus resultierenden ergonomischen und funktionalen Anforderungen zu erfassen, wurden fünf Fokusgruppen zur partizipativen Ideenentwicklung durchgeführt (Möslein et al. 2010). Ein wesentlicher Vorteil gegenüber online- oder strukturierten persönlichen Befragungen ist die direkte Interaktion mit den Teilnehmenden, die eine flexible Diskussion ohne Konzentration auf eine bestimmte Struktur erlaubt (Langford und McDonagh 2003). Der Moderator ist in der Lage, die Teilnehmenden durch die geplanten Phasen zu führen, Folgefragen zu stellen oder Nachfragen zu beantworten. Mögliche Einflussfaktoren auf die Güte der Ergebnisse sind das Management dominanter Gruppenmitglieder, die Qualität der Diskussion und die Erörterung irrelevanter Inhalte. Die Fokusgruppen gliederten sich in drei Hauptbereiche:

1. Ziele und Inhalte eines maritimen Simulationsnetzwerks sowie Erläuterung aktueller Probleme bei Rettungseinsätzen,

2. Zusammenstellung von Anzeige- und Bedienelementen sowie deren Priorisierung in den Kategorien Navigation, Conning (Maschinensteuerelemente) und Kommunikation (sowie weiterer möglicher Kategorien),

\section{Paper Prototyping einer MOS.}

Unter Berücksichtigung der späteren Einsatzhäufigkeit der MOS wurde die Anzahl der Fokusgruppen gewichtet. Die Hauptnutzungsgruppe der Schiffsführer (16) bildete drei Fokusgruppen, Helikopterpiloten und Leitstellenmitarbeiter ergänzten das Probandenkollektiv um jeweils eine weitere Fokusgruppe. Es nahmen insgesamt 31 Personen

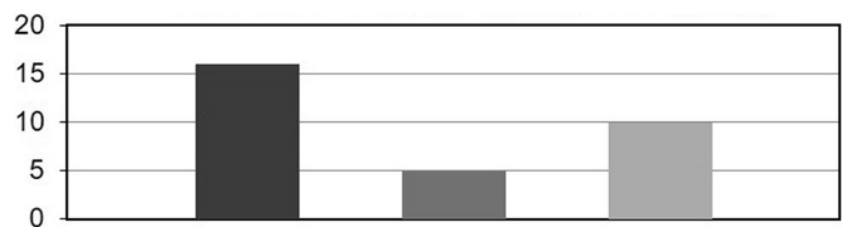

Abb. 2 Anzahl der Teilnehmenden je Nutzergruppe

teil. Die Gruppe der Leitstellenmitarbeiter (10) bestand aus zwei Untergruppen: Leitstellen für Notfälle an Land (1) und Leitstellen für Notfälle auf Wasser (6). Die Fokusgruppe wurde von Mitarbeitern von Offshore-Windparks (3) ergänzt, die aufgrund ihrer immer stärkeren Verbreitung in der Nordsee eine bedeutende Rolle spielen. Die Akquise von Helikopterpiloten gestaltete sich schwierig, jedoch konnten fünf Teilnehmende interessante Hinweise und Beiträge liefern. Die Aufteilung der Teilnehmenden nach Benutzergruppen ist in Abb. 2 dargestellt.

Die Geschlechterverteilung ist mit 29 männlichen und zwei weiblichen Teilnehmenden sehr einseitig, wobei diese Geschlechterverteilung die Grundgesamtheit widerspiegelt (Konstantin 2010). Das Durchschnittsalter aller Teilnehmenden beträgt 48,7 Jahre. Alle Probanden verfügen über viel Erfahrung in ihrer Domäne und haben schon an verschiedenen Realübungen sowie Simulatortrainings teilgenommen, weshalb sie als Lead User einzustufen sind (Bogers et al. 2010).

Alle fünf Fokusgruppen wurden nach dem gleichen Ablaufplan durchgeführt. Nach einer kurzen Willkommensrunde wurden die Agenda und Ziele des Workshops vorgestellt. Anschließend wurden die Themen Simulatorvernetzung und MOS zur Sicherstellung des gleichen Vorwissens aller Teilnehmenden vorgestellt. Um den Gruppensprachfluss zu initiieren, wurden die Teilnehmenden eingeladen, sich selbst, ihren organisatorischen Hintergrund und ihr aktuelles Aufgabengebiet vorzustellen. Anschließend listeten die Teilnehmenden Ziele und Inhalte, die in einem Simu-
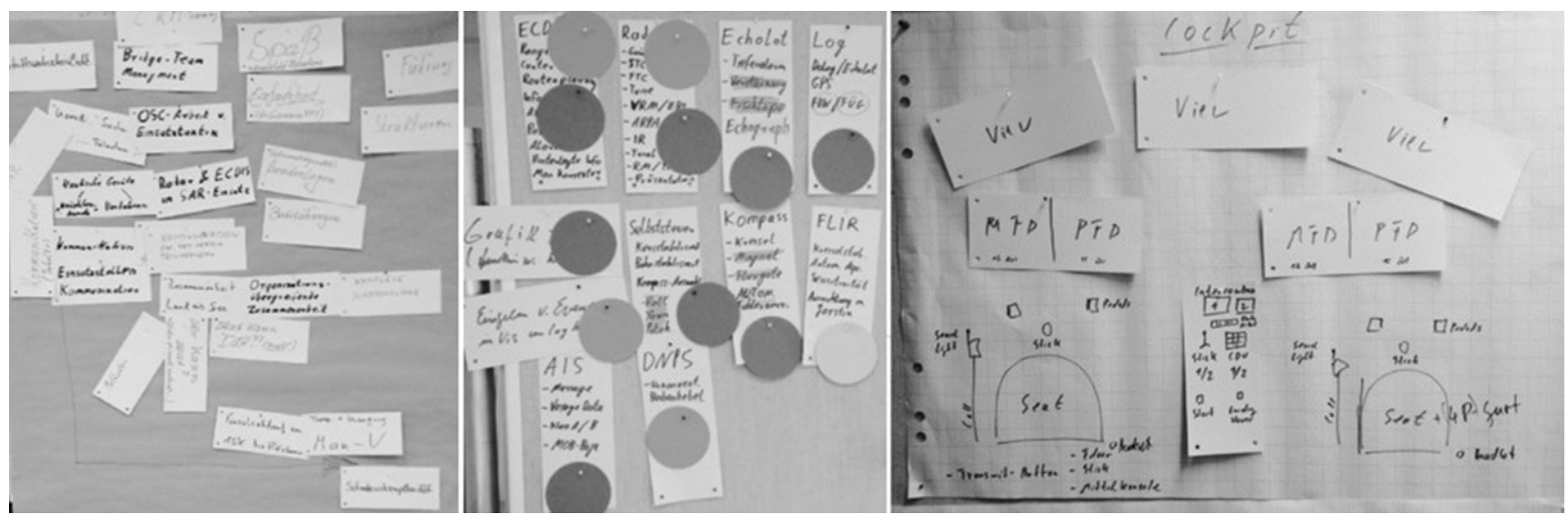

Abb. 3 Von links nach rechts: Ziele und Inhalte eines Workshops mit Schiffsführern, Priorisierte Funktionsauflistung des Bereichs Conning eines Schiffsführerworkshops, Paper Prototyp einer MOS des Workshops mit Helikopterführern 
latornetzwerk trainiert werden sollen, auf. In der folgenden Diskussionsrunde wurden die Ergebnisse besprochen und priorisiert. Nach dem Besuch der vorhandenen Simulatorkabinen im Hauptgebäude der deutschen Gesellschaft zur Rettung Schiffbrüchiger in Bremen, wo alle Fokusgruppen stattfanden, wurden die Teilnehmenden in drei Gruppen eingeteilt. Jede Gruppe war einer von drei Kategorien zugeordnet: Navigation, Conning oder Kommunikation. Innerhalb der Gruppenarbeit wurden alle Funktions-, Anzeigeund Bedienelemente sowie Simulationsparameter erarbeitet. Anschließend wurden die Ergebnisse vor allen Teilnehmenden präsentiert, diskutiert, bei Bedarf ergänzt und in einem dreistufigen System priorisiert. Dabei wurden die Abstufungen ,,muss vorhanden sein“, „kann/sollte vorhanden sein“ und ,wäre schön, wenn vorhanden“ mit unterschiedlich farbigen Punkten markiert. Die Fokusgruppen wurden durch einen Paper-Prototyping-Slot abgeschlossen, in dem die Teilnehmenden ihre eigenen Design-Entwürfe zeichnen bzw. entwerfen konnten. Abschließend wurde eine Zusammenfassung der Ergebnisse und ein Ausblick auf die bevorstehenden Aktivitäten gegeben. Von allen Fokusgruppen wurde eine Audioaufnahme erstellt. Beispielhafte Ergebnisse der Fokusgruppen finden sich in Abb. 3.

\subsection{Ableitung von Anforderungen}

Im Anschluss erfolgte die Auswertung der generierten Daten, wobei zu Beginn der Fokus auf der Zusammenfassung der von den Teilnehmenden genannten Ziele und Inhalte sowie der gewünschten Anzeigen und Bedienelemente lag. Als Grundlage dienten Transkriptionen aller Fokusgruppen. Die insgesamt 490 Seiten Text-Dokumente wurden mittels einer qualitativen Inhaltsanalyse untersucht. Mithilfe von 807 Textstellen entstand ein Kategoriensystem, welches die Anforderungen aus Sicht der Kategorien Mensch, Technik und Organisation an die MOS widerspiegelt. Das Ergebnis stellt ein Katalog mit Anforderungen dar, welcher sich in den simulierten Arbeitsplatz, der Funktionalitätszuweisung (Navigation, Conning, Kommunikation) sowie den Abstufungen muss vorhanden sein, kann/sollte vorhanden sein und wäre schön, wenn vorhanden, unterteilte. Tab. 1 zeigt einen Ausschnitt des Anforderungskatalogs.

Die Ergebnisse zeigen, dass Helikopterpiloten und Schiffsführer in den Bereichen Kommunikation und Navigation einen großen Bereich sich überschneidender Anforderungen aufweisen. Gleichzeitig liegen die Bedürfnisse hinsichtlich der Maschinenüberwachung und -steuerung deutlich auseinander, was jedoch durch eine modulare Bauweise der MOS kompensiert werden kann. Die dritte Endnutzergruppe der Leitstellenmitarbeiter ist mit relativ wenig Aufwand integrierbar, da sie hardwareseitig zur Durchführung ihrer Arbeit lediglich Bildschirme zur Anzeige sowie eine Standard Maus-Tastatur-Kombination als
Tab. 1 Zusammenfassung des Bereichs Navigation über alle Workshops

\begin{tabular}{lll}
\hline Muss vorhanden sein & $\begin{array}{l}\text { Kann/sollte } \\
\text { vorhanden } \\
\text { sein }\end{array}$ & $\begin{array}{l}\text { Wäre schön, } \\
\text { wenn vorhanden }\end{array}$ \\
\hline Radar & $\begin{array}{l}\text { Crewfinder } \\
\text { Luft- bzw. Seefahrkarte }\end{array}$ & $\begin{array}{l}\text { GPS Nr. 2 } \\
\text { Kartentisch }\end{array}$ \\
Kompass & Fernglas & Sextant \\
Geschwindigkeitsanzeige & FLIR & Tidenkalender \\
Höhen- bzw. Tiefenmessgerät & - & - \\
GPS Nr. 1 & & \\
Funkpeiler & & \\
Wetterradar & & \\
Suchscheinwerfer & & \\
Uhr mit Stoppfunktion & & \\
\hline
\end{tabular}

Eingabegerät bevorzugen. In Abb. 4 ist eine Übersicht der Anforderungen an die Arbeitsplätze mit dem Kriterium muss vorhanden sein dargestellt. Die Abkürzungen in Klammern stehen für die jeweilige Nennung durch die einzelnen Nutzergruppen, wobei (S) für Schiffsführer, (H) Helikopterpiloten und (L) für Leitstellenmitarbeiter steht.

\subsection{Designlösungen}

\subsubsection{Festlegung Immersionsgrad}

Im Zuge einer Literaturrecherche bezüglich der Immersionsgradbestimmung und des Präsenzempfinden von Nutzern wurde ermittelt, dass die Immersionsgradfestlegung meist auf Expertenurteilen basiert und nicht objektiv messbzw. nachvollziehbar ist. Lediglich Präsenz kann als persönliche Wahrnehmung erhoben werden. Sie wird definiert als Erfahrung in einer virtuellen Umgebung, woanders zu sein als an dem Ort, an dem sich die Person physisch befindet. Das Präsenzempfinden schließt dabei eine willentliche Beseitigung von Zweifeln bezüglich des Ortes, an dem sich die Person physisch befindet, ein (Slater und Usoh 1993, Slater et al. 1994).

Im Rahmen eines Expertenworkshops wurde mit sechs Teilnehmenden diese Thematik aufgegriffen und praxisnah diskutiert. Die Experten stellten fest, dass die Technologie nur ein Faktor der Wahrnehmung von Präsenz ist. Erfahrungswerte einer Vielzahl durchgeführter Übungen zeigen, dass die intrinsische Motivation der Teilnehmenden deren Präsenzempfinden stark beeinflusst. Hierbei wurde von den Experten festgehalten, dass die Akzeptanz von realen oder simulierten Gerätschaften von den $\mathrm{zu}$ trainierenden Übungszielen abhängt. Weiterhin wurde vor allem der „Spieltrieb“ bei monotonen Situationen als Ablenkung und damit Störgröße identifiziert. In Folge der Literaturrecherche und des Expertenworkshops wurde als Anforderung an 


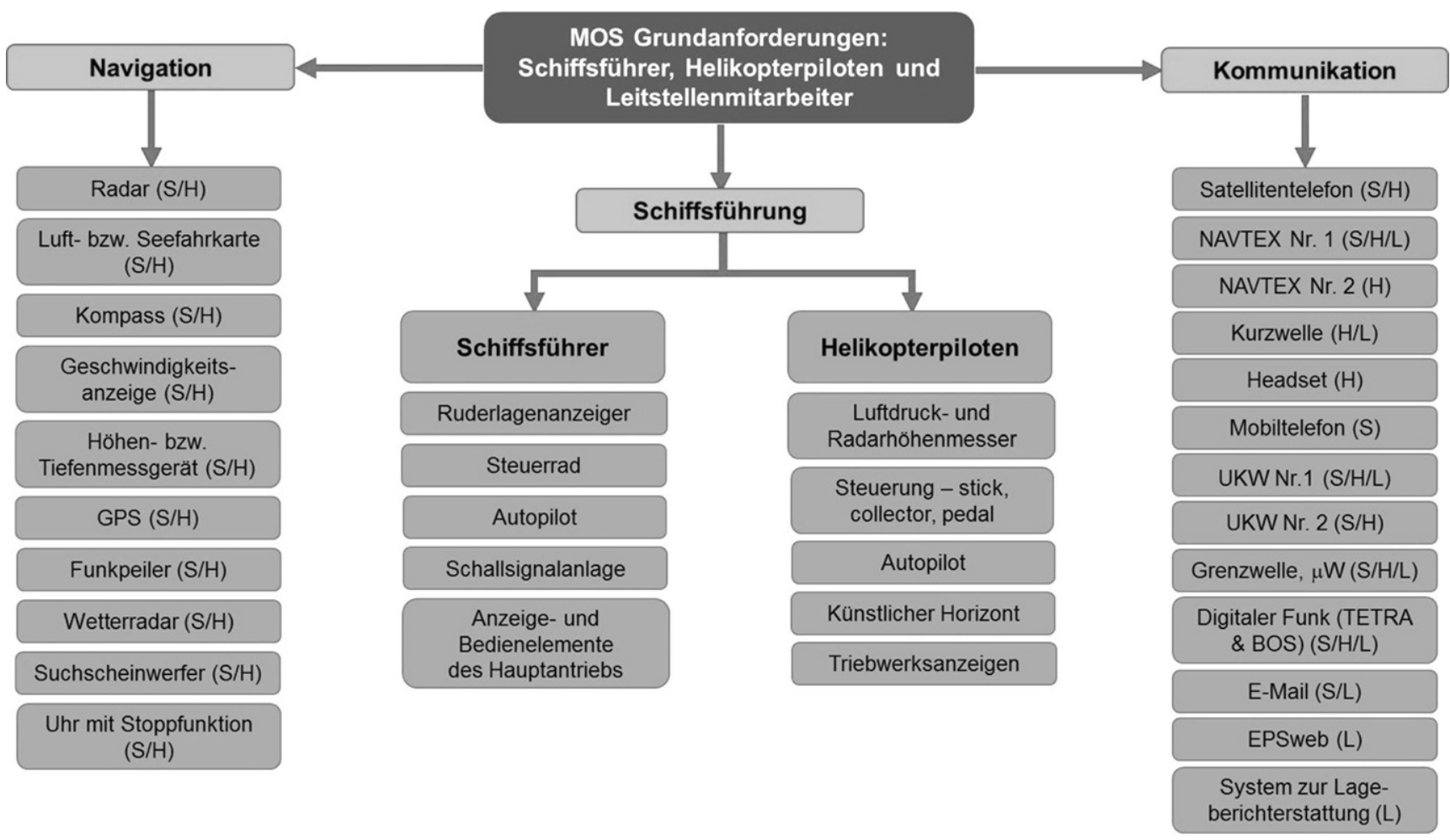

Abb. 4 Grundanforderungen an die MOS über alle Nutzergruppen (S - Schiffsführer, H - Helikopterpiloten, L - Leitstellenmitarbeiter)
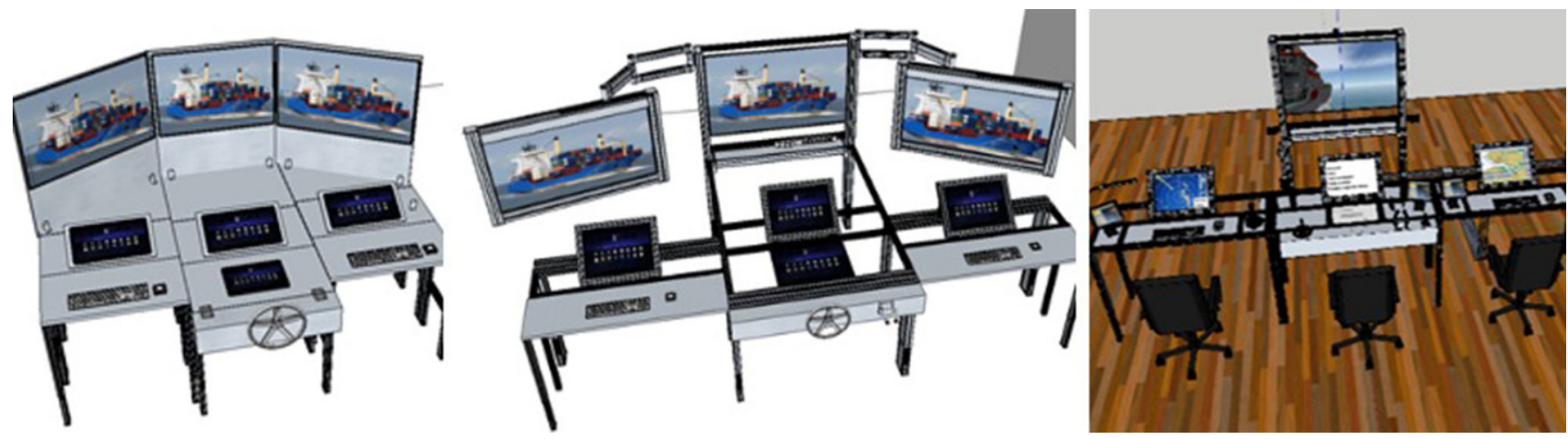

Abb. 5 Vergleich des Schiffsführerarbeitsplatzes über alle drei Iterationen (von links nach rechts erste bis dritte Iteration)

die Prototypen der MOS (siehe Abb. 5) ein niedriger Immersionsgrad in Form eines geringen Realitätsgrads festgelegt. Als Basis für die weiteren Entwicklungsarbeiten sollen daher weniger realitätsgetreue Stellteile genutzt sowie Arbeitsumgebungen realisiert werden.

\subsubsection{Design der MOS}

Dem nutzerzentrierten Entwicklungsprozess (ISO 2010) folgend, schloss sich die Gestaltung der Schiffsführer-, Helikopter- und Leitstellenarbeitsplätze in verschiedenen Reifegradstufen an. Erste Entwürfe basierten auf Paper-Prototyping-Skizzen, welche im Rahmen der Fokusgruppen zur Anforderungsanalyse erstellt wurden. Für eine schnelle
Umsetzung wurde das Programm Microsoft Visio gewählt. Darauf aufbauend erfolgten Gestaltungsentwürfe mittels der Software SketchUp des Herstellers Trimble. Hiermit konnten dreidimensionale Abbildungen der Arbeitsplätze geschaffen werden, welche einen hohen Detailgrad ermöglichten.

Der anschließende Gestaltungsprozess verlief iterativ, wobei die oben beschriebene Prototyping-Lösung als Basis diente. In Anlehnung an die Methodik zur Arbeitsplatzplanung (Kleinhenz 2011) wurde dabei zuerst nach Standardkomponenten für Flächenmodule recherchiert, welche für den Grobaufbau in den Prototypen genutzt werden konnten. Unter anderem wurden höhenverstellbare Standardtische sowie eine Monitorhalterung zusammengestellt. 
Zudem finden sich Einzeltische, welche einen variablen Anstellwinkel der Bildschirme ermöglichen, in allen drei Arbeitsplätzen wieder. Danach wurden die Flächenmodule auf Basis möglicher Arbeitsabläufe in eine Flächenstruktur überführt. Zuletzt wurde die resultierende Anordnung der Einzelkomponenten nach ihrer Praktikabilität und der Erfüllung der Anforderungen bewertet und daraus resultierende Änderungen umgesetzt. Die resultierenden Arbeitsplätze bildeten die zweite Iteration.

Innerhalb der dritten Iteration erfolgte eine Anpassung der spezifischen Details der Arbeitsplätze. Hierfür wurde auf die Ergebnisse der qualitativen Inhaltsanalyse zurückgegriffen, um den Nutzeranforderungen an die Arbeitsplätze bestmöglich gerecht zu werden. Im Detail wurden weitere Elemente zur Aufgabenerfüllung, wie Drucker, Kartentische, Kommunikationseinheiten und Headsets, an die Arbeitsplätze angeordnet. Weiterhin erfolgte die Zuordnung der einzelnen Softwarekomponenten zu den Bildschirmen sowie beim Helikopterarbeitsplatz die Anordnung von Anzeigen der Primary Flight Displays. Die beschriebene Vorgehensweise soll anhand der Gegenüberstellung der Iterationen in Abb. 5 verdeutlicht werden.

\subsection{Designevaluation}

In der finalen Phase des nutzerzentrierten Entwicklungsprozesses wurde nach der Gestaltung der Prototypen ein Usability-Test in Form einer Nutzerevaluation vorgenommen (Sarodnick und Brau 2006). Hierbei wurde auf LeadUser zurückgegriffen, welche im Rahmen der vorher erfolgten Fokusgruppenworkshops bereits an der Anforderungserstellung mitgewirkt haben. Aufgrund einer geringen Teilnehmendenquote wurden zudem weitere Personen von, im Projekt beteiligten Organisationen kontaktiert. Aufgrund der eingeschränkten Erreichbarkeit der Teilnehmenden sowie einer geringen zeitlichen Flexibilität wurden die Usability-Tests mit teilstrukturierten telefonischen Interviews durchgeführt. Dabei wurde ein Methodenmix aus qualitativen und quantitativen Daten angewandt. Hierfür erhielten die Teilnehmenden im Vorfeld der Befragung ein 3DPrototyp der jeweiligen MOS, welcher sich mit dem Adobe Acrobat-Reader öffnen und betrachten ließ. Im Rahmen der Interviews sollten sich die Befragten in verschiedene Situationen eines Simulatortrainings versetzen, wofür ihnen mittels eines Story Boards eine kurze Geschichte eines Einsatzes beschrieben wurde (Richter und Flückiger 2013). Anschließend erfolgte die Abfrage von Elementen, welche im 3D-Prototyp noch fehlen oder anders positioniert werden sollten. Weiterhin wurden den Probanden Fragen bezüglich deren Beurteilung zur organisatorischen Umsetzung des Simulatortrainings mit dem präsentierten Prototyp gestellt. Abschließend wurden der User Experience Questionnaire (UEQ, Laugwitz et al. 2008) genutzt, um die Usability und

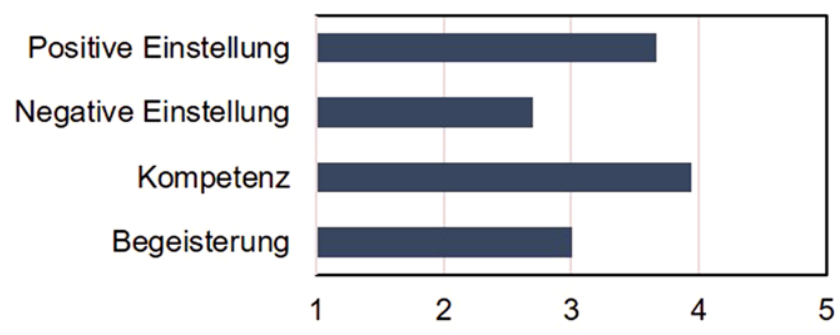

Abb. 6 Technikaffinität der Probanden (1 geringe bis 5 hohe Übereinstimmung)

Zufriedenstellung zu bewerten. Diese Fragebögen wurden den Teilnehmenden zusammen mit dem Prototyp im Voraus per E-Mail zugesandt.

Im Rahmen der Erhebung konnten Bewertungen von insgesamt 15 männlichen Probanden aufgenommen werden. Das durchschnittliche Alter betrug 50,8 Jahre ( $\mathrm{SD}=9,8$ ). Weiterhin wiesen die Nutzer für den Bereich der Leitstellen eine Erfahrung von 17,7 Jahren ( $\mathrm{SD}=12,0)$, für den Bereich Helikopterpilot 23,6 Jahren (SD=10,4) und für den Bereich Schiffsführer eine Erfahrung von 12,1 Jahren $(S D=7,8)$ auf. Dabei weist die Stichprobe eine hohe Technikaffinität auf, welche mittels des Fragebogens TA-EG (Karrer et al. 2009) erfasst wurde und in Abb. 6 dargestellt ist.

Da ein statischer 3D-Prototyp genutzt wurde, wurde beim UEQ auf die Subskalen Durchschaubarkeit, Effizienz und Vorhersagbarkeit verzichtet und lediglich die Subskalen Attraktivität, Stimulation und Originalität erhoben. Im Rahmen der Auswertung sind Werte, welche $-0,8$ unterschreiten beziehungsweise $+0,8$ überschreiten, als aussagekräftig einzustufen (siehe blaue Linien in Abb. 7, Laugwitz et al. 2008). Die Ergebnisse sind in Abb. 7 zusammengefasst.

Die Ergebnisse der UEQ-Bewertungen decken sich mit den Anmerkungen der Nutzer aus den teilstrukturierten Interviews. Während Schiffsführer- und HelikopterpilotenArbeitsplätze bis auf die Originalität besonders positiv bewertet wurden, erzielte der Leitstellenarbeitsplatz im Mittel eine neutrale Bewertung. In der Auswertung der Interviews konnte ermittelt werden, dass vor allem der geringe Platz zum Arbeiten mit Stift und Papier und die zu geringe Anzahl an Displays kritisch betrachtet wurden. Für die weiteren Arbeitsplätze wurden Hinweise zum Ändern der Position oder Ausrichtung einzelner Objekte aufgenommen. Ebenso konnten Hinweise bezüglich des Auf- und Abbaus, wie die Einführung von Farbkodierungen und Poka-YokeElementen, aufgenommen werden. In Abb. 8 wird das anhand des nutzerzentrierten Entwicklungs-prozesses entstandene Ergebnis des Leitstellenarbeitsplatzes abgebildet.

Im Gegensatz zu dem Leitstellenarbeitsplatz werden für die weiteren Arbeitsplätze neben Kommunikationsanforderungen (communication) noch Anforderungen an Navigation (navigation) und die Kommandoeinheit (conning) ge- 
Abb. 7 UEQ-Bewertung für jede MOS Spezifikation (Schiffsführer, Helikopterpiloten und Leitstellenmitarbeiter)
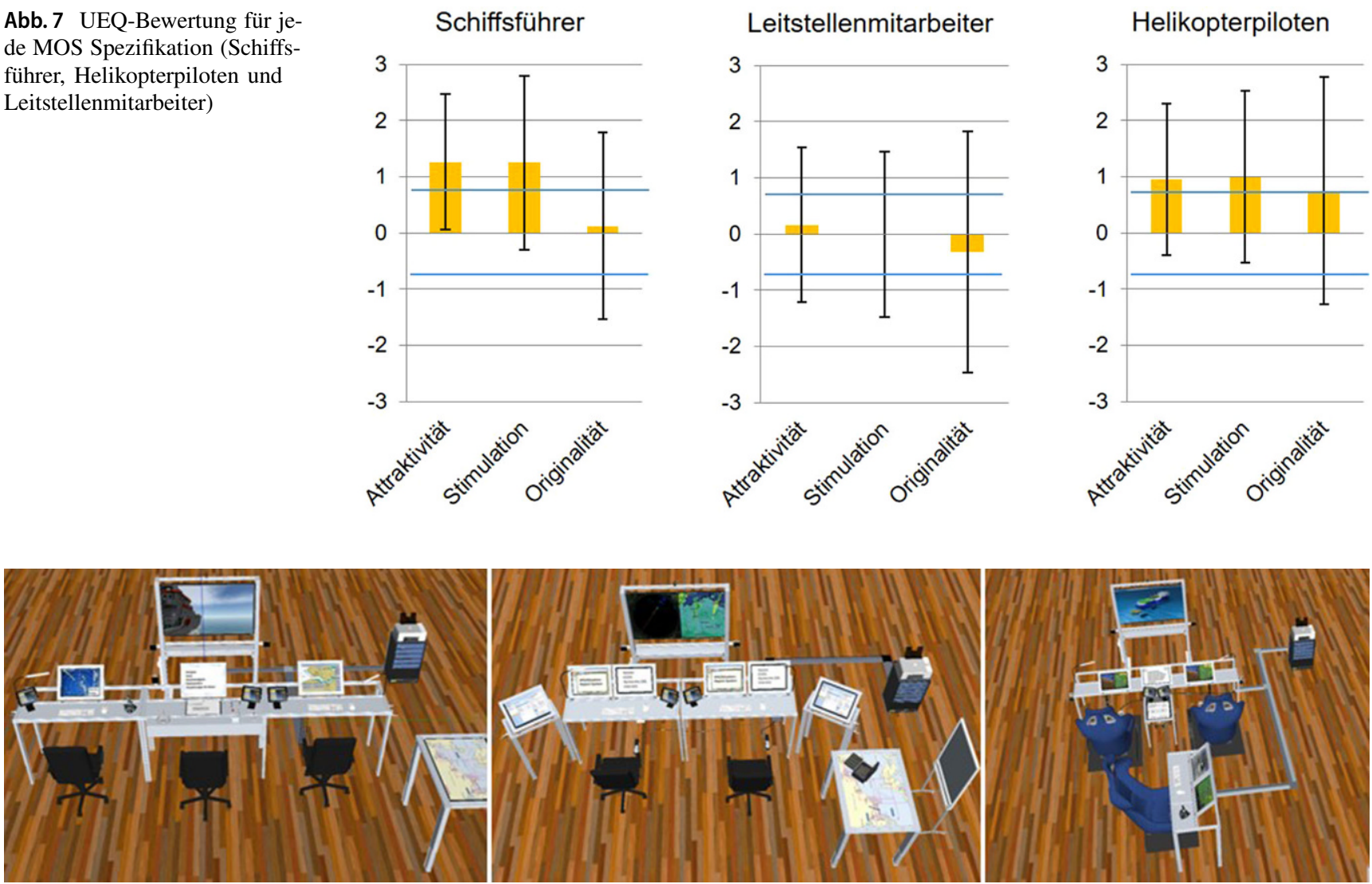

Abb. 8 MOS Spezifikation für Schiffsführer (li.), Leitstellenmitarbeiter (mi.) und Helikopterpiloten (re.)

stellt. Eine Besonder-heit bei der Gestaltung des Helikopterarbeitsplatzes lag in dem Widerspruch zwischen High-EndFlugsimulator und mobilem multifunktionalen Simulator. Der unter den Entwicklungspartnern erzielte Kompromiss für eine abstrakte und wenig detail-, aber funktionsreiche Umsetzung wurde mit entsprechend doppelt verwendeten Elementen und möglichst wenig physischen Stellteilen umgesetzt.

Letztlich wurde speziell für den Schiffsführerarbeitsplatz eine conning-Einheit (Modul Schiffsführer) entworfen. Diese beinhaltet die spezifischen Stellteile des Schiffsführers für Navigationsaufgaben. Sie ist als separates Modul nicht multifunktional ausgelegt, da die Stellteile mehrfach verwendbare Funktionalitäten ausschließen. Abb. 8 stellt eine Gesamtübersicht für den Arbeitsplatz dar.

Neben den hardwareseitigen Anforderungen ist ebenfalls die Anordnung der Softwarebestandteile von Interesse. Hierbei wurde im Rahmen der 3D-PDFs eine Anordnung der Anwendungen vorgeschlagen, welche von den Nutzern akzeptiert wurde und den Anforderungen entspricht. Jedoch wurde mehrfach der Wunsch nach einer individuellen Platzierung der Elemente geäußert. Diese sollte für den Arbeitsplatz der Leitstellenmitarbeiter und Schiffsführer umgesetzt werden. Für den Arbeitsplatz der Helikopterpiloten ist eine selbstständige Anordnung der Anwendungen nicht zu empfehlen, da sich diese an den vorhandenen Systemen orientiert und somit standardisiert ist. Zudem ist die Anzahl an Anzeigeelementen wesentlich höher, weshalb das Dialoggestaltungskriterium der Fehlertoleranz wesentlich geringer wäre im Vergleich zu den anderen Systemen.

\section{Implikationen, Limitationen und Ausblick}

Im Beitrag wurden die ergonomische Gestaltung und Weiterentwicklung der MOS mithilfe des nutzerzentrierten Entwicklungsprozesses (ISO 2010) dargelegt. Auf Basis von Fokusgruppen mit zukünftigen Nutzenden konnte ein erstes Design entworfen werden, das als Grundlage für weitere Entwicklungsarbeiten diente. Nach der abschließenden Nutzerevaluation entstand der finale Prototyp, der die Anforderungen an ein kompaktes und reduziertes Hardwaredesign erfüllte und zudem eine hohe Gebrauchstauglichkeit aufwies.

3.1. Inhaltliche Implikationen Die Anforderungsanalyse zeigte, dass die Bedürfnisse der drei zukünftigen Nutzergruppen in Abhängigkeit der auszuführenden Aktivität sehr 
weit streuen. Überschneidungen in allen drei Nutzergruppen ließen sich nur im Bereich Kommunikation feststellen. In der Navigations-Domäne sind ähnliche Anforderungen zwischen den beiden Gruppen Schiffsführer und Helikopterpiloten zu verzeichnen.

Insbesondere im Bereich der zu integrierenden Anzeigen und Stellteile zur Steuerung (conning) sind große Differenzen aufgeführt worden (siehe Abb. 4 und Abb. 8). Dies bestätigte das Entwicklungsziel einer modularen Bauweise. Durch die Entwicklung verschiedener Module für den Mittelbereich der MOS konnte den verschiedenen Anforderungen gerecht werden.

Die abschließende Evaluierung zeigte, dass die Prototypen in den Subskalen Attraktivität, Stimulation und Originalität des UEQ ein zufriedenstellendes Ergebnis liefern und somit eine gute Basis für weiterführende Entwicklungen darstellen (Laugwitz et al. 2008).

Organisationsübergreifende Trainingsszenarien können nach der präsentierten Entwicklung einer MOS mit einfachen, modularen und vernetzten Simulatoren umgesetzt werden, was die Annahme von Riener (2010) bestätigt. Steht die Zusammenarbeit und Kommunikation während einer maritimen Großschadenslage im Mittelpunkt des Trainings, kann in Bestätigung von bestehenden Studien (Wright und Madey 2008) darüber hinaus auf Simulatoren verzichtet werden, die auf einen konkreten Anwendungsfall zugeschnitten sind.

3.2 Methodische Implikationen Die Kombination aus quantitativen und qualitativen Methoden erwies sich sowohl auf Probanden- als auch auf Forscherseite als geeignet und bestätigt bzw. bereichert damit bestehende Kenntnisse (Li und Earnest 2015). Sowohl den beteiligten, im Anwendungsgebiet teilweise mit wenig Hintergrundwissen ausgestatteten Wissenschaftlern als auch den Teilnehmenden, die nur rudimentäre Kenntnisse bzgl. mobiler und ergonomisch gestalteter Simulatoren vorzuweisen hatten, half der Methodenmix beim Einstieg in das Themengebiet.

Dabei hat sich insbesondere der Einsatz von Fokusgruppen während der ersten Phase des nutzerzentrierten Entwicklungsprozesses in diesem Anwendungskontext als passend erwiesen. Die Möglichkeit der Moderation und Lenkung der Diskussion sowie die direkte Interaktion mit den Probanden erlaubte außerdem tiefe Einblicke in die unterschiedlichen Einsatzdomänen der MOS und deren spezifische Problembereiche innerhalb komplexer Schadenslagen auf See.

Der abschließende Paper-Prototyping-Prozess stellte eine sehr breite Basis für erste Designlösungen der MOS dar. Dieses Vorgehen wird vor allem für Produktentwickler empfohlen, die sich in einer neuen Anwendungsdomäne bewegen und einen geringen Kenntnisstand des Untersuchungsobjektes vorweisen sowie gleichzeitig erste Ideen- generierungen durchführen wollen (Töpfer und Silbermann 2008). Die Erarbeitung der Papierprototypen für die MOS bestätigte diese Empfehlung.

Als Basis für die ersten virtuellen Prototypen, welche anschließend in zwei weiteren Entwicklungsschleifen verbessert werden konnten, dienten die Papierprototypen. Die methodische Kombination der Versendung von 3D-Prototypen, die im Vorlauf durch die Probanden betrachtet werden konnten, und anschließender Fragebogen-untersuchung sowie Interviewanwendung zeigte sich als besonders geeignet für Evaluationen, in denen sich Probanden und Versuchsleitung in großer räumlicher Entfernung befinden bzw. gemeinsame Workshops aus organisatorischen Gründen nur sehr schwer durchführbar sind.

3.3 Limitationen und Ausblick Auch für die MOS gilt, dass ein Produkt, das anhand des UCD entwickelt wurde, keinen „Erfolg“ garantiert (Ritter et al. 2014). Eine hohe Gebrauchstauglichkeit der MOS führt nicht automatisch zu hohem Lernerfolg, weil dieser von vielen verschiedenen Faktoren (siehe 2.3.1) abhängig ist. Darüber hinaus sichert eine hohe Gebrauchstauglichkeit auf Mikroebene keine genaue Passung zu allen auf unterschiedlichen Schiffen und Helikoptern verfügbaren Anzeige- und Bedienelementen. Dies kann trotz höherer Usability der simulierten Umgebung zu erwartungskonformitätsbedingten Fehlbedienungen führen (Baxter et al. 2005).

Die im Verlauf dieses Entwicklungsprozesses erarbeiteten finalen (virtuellen) Prototypen wurden nach Abschluss in reale MOS umgesetzt. Um deren Grad an Ergonomie und erwartungsgerechter Handhabung zu untersuchen, sollten sie Gegenstand von Probandenstudien werden. Im Fokus dieser Studien sollte die Interaktion mit der MOS und deren Wirkung auf den Menschen stehen, da die Usability der Anzeige- und Bedienelemente im Rahmen der Bewertung der digitalen Prototypen nicht untersucht werden konnte. Darüber hinaus dient ein solcher Test der Überprüfung der Modularisierbarkeit der MOS, um eine zu anwendungsfallspezifische Entwicklung auszuschließen und eine hohe Generalisierbarkeit zu gewährleisten (McLoone et al. 2010). Gleichzeitig erscheint eine Kontrolle der Realitätsnähe der Online-Prototypenevaluation sinnvoll, um die Auswahl der gestellten Arbeitsaufgaben und Situationsmerkmale zu validieren und dömanenübergreifende Aussagen zur Anwendung von Online-Evaluationen abzuleiten (Scholtz 2004). Weiterhin konnte im Rahmen der Online-Evaluation nicht untersucht werden, wie effizient die Kommunikation zwischen den Teilnehmern in den MOS nachgebildet wird. Dies muss in der Evaluation der realen MOS geschehen. Abschließend sollten Lernerfolgsmessungen durchgeführt und mit Erfahrungen aus realen Übungen in Verbindung gesetzt werden, um die Wirksamkeit einer Simulator Übung weiter $\mathrm{zu}$ validieren. 
Funding Open Access funding provided by Projekt DEAL.

Open Access Dieser Artikel wird unter der Creative Commons Namensnennung 4.0 International Lizenz veröffentlicht, welche die Nutzung, Vervielfältigung, Bearbeitung, Verbreitung und Wiedergabe in jeglichem Medium und Format erlaubt, sofern Sie den/die ursprünglichen Autor(en) und die Quelle ordnungsgemäß nennen, einen Link zur Creative Commons Lizenz beifügen und angeben, ob Änderungen vorgenommen wurden.

Die in diesem Artikel enthaltenen Bilder und sonstiges Drittmaterial unterliegen ebenfalls der genannten Creative Commons Lizenz, sofern sich aus der Abbildungslegende nichts anderes ergibt. Sofern das betreffende Material nicht unter der genannten Creative Commons Lizenz steht und die betreffende Handlung nicht nach gesetzlichen Vorschriften erlaubt ist, ist für die oben aufgeführten Weiterverwendungen des Materials die Einwilligung des jeweiligen Rechteinhabers einzuholen.

Weitere Details zur Lizenz entnehmen Sie bitte der Lizenzinformation auf http://creativecommons.org/licenses/by/4.0/deed.de.

\section{Literatur}

Bailey, B.; Biehl, J.; Cook, D.; Metcalf, H.: Adapting paper prototyping for designing user interfaces for multiple display environments. In: Personal and Ubiquitous Computing, 12(3), 269-277, 2007

Baxter GD, Monk AF, Tan K, Dear PRF, Newell SJ (2005) Using cognitive task analysis to facilitate the integration of decision support systems into the neonatal intensive care unit. Artif Intell Med $35: 243-257$

Bevan N (2009) International standards for usability should be more widely used. J Usabil Stud 4:106-113

Blackmon MH (2004) Cognitive walkthrough. In Bainbridge WS (Hrsg) Encyclopedia of Human-Computer Interaction, Bd. 1. Berkshire, Great Barrington, S 104-107

Bogers M, Afuah A, Bastian B (2010) Users as innovators: A review, critique, and future research directions. J Management 36(4):857-875

Buchenau S (2020) Feuerwehr Einsätze in Häfen, auf und am Wasser - Die größten Schiffskatastrophen im neuen. Jahrtausend In: Feuerwehr-magazin Ebner Media Group Gmbh Co Kg. https://www. feuerwehrmagazin.de/wissen/die-groessten-schiffskatastrophenim-neuen-jahrtausend-78709. Zugegriffen: 12.04.2020

Chammas A, Quaresma M, Mont'Alvão C (2015) A Closer Look on the User Centred Design. Procedia Manuf 3:5397-5404

Creswell JW (2009) Research Design. Qualitative, quantitative, and mixed methods approaches, 3. Aufl. SAGE, Thousand Oaks

Ecker M (2016) Usability und usability engineering zur Gestaltung von Lernsystemen - User-centered Methoden zur nachhaltigen Entwicklung digitaler Bildungsangebote. Technischer Bericht 1/2015, Pädagogische Hochschule Weingarten, S 1-38

Flin RH, O'Connor P, Crichton M (2008) Safety at the sharp end: A guide to non-technical skills. CRC, Taylor \& Francis Group, Boca Raton

Hofinger G (2008) Teamtrainings für Krisenbewältigung. In: Buerschaper, C.; Starke, S.: Team und Führung in kritischen Situationen. Verlag für Polizeiwissenschaft, Frankfurt am Main, S $190-205$

International Organization for Standardization: Ergonomics of humansystem interaction-Part 210: Human-centred design for interactive systems, 2010

Kahraman, Z. E. H.: Using user-centered design approach in course design. Procedia-Social and Behavioral Sciences, 2(2), 2071-2076, 2010
Karrer K, Glaser C, Clemens C, Bruder C (2009) Technikaffinität erfassen - der Fragebogen TA-EG. Der Mensch im Mittelpunkt technischer. Systeme 8:196-201

Kleinhenz S (2011) Der Büroarbeitsplatz: Handbuch für die Gestaltung von Arbeitsplätzen im Büro. Haefner, Heidelberg

Konstantin F (2010) Seenotrettung: Eine Frau hat das Sagen. In: Flensburger Tageblatt. https://www.shz.de/lokales/flensburgertageblatt/seenotrettung-eine-frau-hat-das-sagen-id2442341.html. Zugegriffen: 20.03.2019

Langford J, McDonagh D (2003) Focus Groups: Supporting effective product development. Taylor \& Francis, London

Laugwitz B, Held T, Schrepp M (2008) Construction and evaluation of a user experience questionnaire. In: Symposium of the Austrian HCI and Usability Engineering Grou. Springer, Berlin, Heidelberg, S 63-76

Li J, Earnest J (2015) Das Beste aus zwei Welten - Vorteile einer Kombination von qualitativen und quantitativen. Forschungsmethoden In: Wzb Mitteilungen 150:30-33

Lübcke T, Steigenberger N, Bornhorst C, Roßner P, Kemna J (2015) From Ship Handling to Maritime Incident Response Simulation-Advanced Networked Simulation. MARSIM. conference, Bd. 2015. Newcastle, UK

Maguire M (2001) Methods to support human-centred design. Int J Human-computer Stud 55:587-634

McLoone E, Jacobson M, Hegg C, Johnson PW (2010) User-centered design and evaluation of a next generation fixed-split ergonomic keyboard. Work 37(4):445-456

Molka-Danielsen, J.; Prasolova-Førland, E.; Fominykh, M.; Lamb, K. Use of a Collaborative Virtual Reality Simulation for Multi-Professional Training in Emergency Management Communications. 2018 IEEE International Conference on Teaching, Assessment, and Learning for Engineering (TALE), Wollongong, NSW, 2018, 408-415, 2018

Möslein KM, Halle JBA, Bullinger AC (2010) Open Evaluation: IT für das Innovations-management. Wirtschaftsinformatik Manag. https://doi.org/10.1007/BF03248287

Nielsen, J.: Enhancing the explanatory power of usability heuristics. Proc. ACM CHI'94 Conf., Boston, MA, April 24-28, 152-158, 1994

Richter M, Flückiger MD (2013) Usability Engineering kompakt: benutzbare Produkte gezielt entwickeln. Springer, Berlin

Riener A (2010) Simulating On-the-road Behaviour Using Driving Simulators. In: Proceedings or the 3rd International Conference on Advances in Computer-Human Interactions. IEEE Computer Society Press, Saint Maarten

Ritter FE, Baxter GD, Churchill EF (2014) Introducing User-Centered Systems Design. In: Foundations for Designing User-Centered Systems. Springer, London, S 3-31

Rosenkötter S (2015) Fehmarn - Das Drama um die Lisco Gloria. https://www.ln-online.de/Lokales/Ostholstein/Das-Drama-umdie-Lisco-Gloria. Zugegriffen: 16.07.2019

Salas E, Wilson KA, Burke CS, Wightman DC (2006) Does Crew Resource Management Training Work? An Update, and Extension, and Some Critical Needs. Hum Factors 48(2):392-412

Sarodnick F, Brau H (2006) Methoden der usability evaluation. Huber, Bern

Schlamp HJ (2018) Schiffshavarie: Was wurde aus der „Costa Concordia“ und aus Kapitän Schettino? Abgerufen 16.07.19, von. https://www.spiegel.de/panorama/costa-concordia-was-wurdeaus-dem-ungluecksschiff-a-1183275.html. Zugegriffen: 16.07.2019

Schlick CM, Bruder R, Luczak H (2010) Arbeitswissenschaft. Springer, Berlin

Scholtz J (2004) Usability Evaluation. In National Institute of Standards and Technology, Gaithersburg, Maryland. https://doi.org/ 10.1109/HSI.2016.7529638 
Shneiderman B, Plaisant C, Cohen M, Jacobs S, Elmqvist N (2016) Designing the user interface. Strategies for effective human-computer interaction, 6. Aufl. Pearson, Harlow, England

Slater M, Usoh M (1993) Representations systems, perceptual position, and presence in immersive virtual environments. Presence: Teleoperators Virtual Environ 2(3):221-233

Slater M, Usoh M, Steed A (1994) Depth of presence in virtual environments. Presence: Teleoperators Virtual Environ 3(2):130-144

Still B, Morris J (2010) The Blank-Page Technique: Reinvigorating Paper Prototyping in Usability Testing. IEEE Trans Profess Commun 53(2):144-157

Strohschneider S (2012) Human Factors Training. In: Badke-Schaube, P. et al., Human Factors: Psychologie sicheren Handelns in Risikobranchen. Springer, Berlin

Tena-Chollet F, Tixier J, Dandrieux A, Slangen P (2016) Training decision-makers: Existing strategies for natural and technological crisis management and specifications of an improved simulationbased tool. Safety Sci 97:144-153
Töpfer A, Silbermann S (2008) Einsatz von Kunden-Fokusgruppen. In: Handbuch Kundenmanagement. Springer, Berlin, Heidelberg, S 267-279

Weick KE (2010) Reflections on Enacted Sensemaking in the Bhopal Disaster. Jour of Manage Stud 47(3):537-550

Wright TE, Madey G (2008) A prototype virtual emergency operations center using a collaborative virtual environment. 5th International ISCRAM Conference, Washington, USA. ISCRAM Digital Library, Ocean City

Zoller G (2014) Kommunikation und Kooperation zwischen ausgewählten Organisationen der Behörden und Organisationen mit Sicherheitsaufgaben bei größeren Einsatzlagen - Erfolgskritische Faktoren des. Prozessmanagements Diss Bergische Univ Wupp. urn:nbn:de:hbz:468-20140908-123736-9. Zugegriffen: 20.01.2020 